\title{
Spectral analysis of gluonic pole matrix elements for fragmentation
}

\author{
L. P. Gamberg* \\ Division of Science, Penn State University Berks Campus, Reading,Pennsylvania 19610, USA \\ A. Mukherje母 \\ Physics Department, Indian Institute of Technology, Powai, Mumbai 400076, India \\ P.J. Mulder团 \\ Department of Physics and Astronomy, VU University \\ NL-1081 HV Amsterdam, the Netherlands
}

(Dated: November 1, 2018)

\begin{abstract}
The non-vanishing of gluonic pole matrix elements can explain the appearance of single spin asymmetries in high-energy scattering processes. We use a spectator framework approach to investigate the spectral properties of quark-quark-gluon correlators and use this to study gluonic pole matrix elements. Such matrix elements appear in principle both for distribution functions such as the Sivers function and fragmentation functions such as the Collins function. We find that for a large class of spectator models, the contribution of the gluonic pole matrix element in fragmentation functions vanishes. This outcome is important in the study of universality for fragmentation functions and confirms findings using a different approach.
\end{abstract}

\section{INTRODUCTION}

In high-energy scattering processes the structure of hadrons is accounted for using quark and gluon correlators, forward matrix elements of non-local quark and gluon operators between hadronic states. Making an expansion in the (inverse) hard scale, the relevant component of the momentum of partons (quarks and gluons) is the one collinear to hadrons and correspondingly the non-locality in the matrix elements is restricted to the light-cone. Moreover, all leading dynamical effects come from two-field configurations at two light-like separated points, which are easily interpreted as parton densities or parton decay functions [1, 2]. These are the parton distribution functions depending on the momentum fraction $x$ relating the parton momentum $k=x P$ to the hadron momentum $P$ or the fragmentation functions of partons into hadrons depending on the momentum fraction $z$, relating the parton momentum $k$ and the hadron momentum $P=z k$.

At sub-leading order in the hard scale or when explicitly measuring transverse momenta, other matrix elements become important such as the three-parton correlators containing parton fields at three different spacetime points with light-like separations and two-parton correlators with also transverse separation (light-front correlations). These latter (light-front) correlators are described in terms of transverse momentum dependent (TMD) distribution and fragmentation functions, which are sensitive to the intrinsic transverse momenta of partons in hadrons, $k=x P+k_{T}$ in a frame in which the

\footnotetext{
*Electronic address: $\operatorname{lpg} 10 @$ psu.edu

${ }^{\dagger}$ Electronic address: asmita@phy.iitb.ac.in

‡Electronic address: mulders@few.vu.nl
}

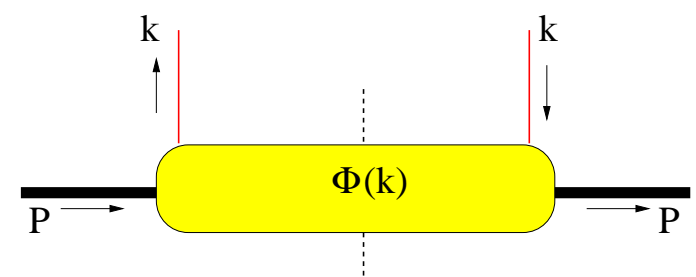

(a)

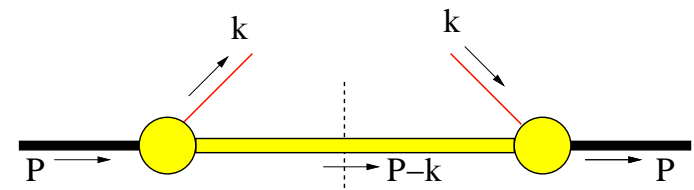

(b)

FIG. 1: The graphical representation of the quark-quark correlator in the case of distributions of partons with momentum $k$ in a hadron with momentum $P$ (a) and the spectator model description (b).

hadron does not have transverse momentum $\left(P_{T}=0\right)$ or for fragmentation $k=\frac{1}{z} P+k_{T}$. In this case one often refers to the hadron transverse momentum $P_{\perp}=-z k_{T}$ (in a frame in which the parton does not have a transverse momentum $\left.\left(k_{\perp}=0\right)\right)$.

In this paper, we will investigate multi-parton correlators with one additional gluon in which the zeromomentum limit will be studied [3, 44, 5, 6, 7, 8, 9, 10]. These are so-called gluonic pole matrix elements or Qiu-Sterman matrix elements, that have opposite timereversal ( $\mathrm{T}$ ) behavior as compared to the matrix elements without the gluon. Such matrix elements involving timereversal odd (T-odd) operator combinations are of interest because they are essential for understanding single 


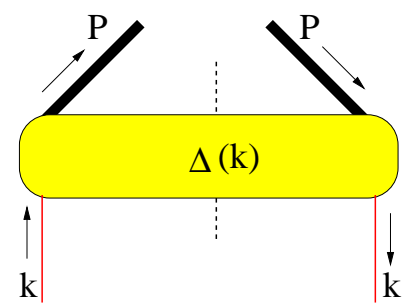

(a)

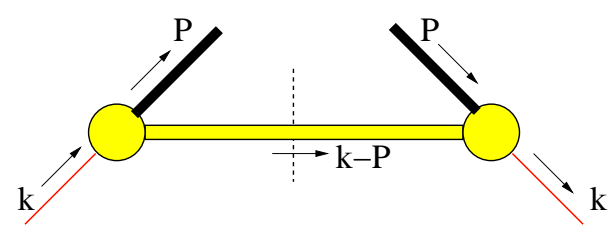

(b)

FIG. 2: The graphical representation of the correlator in the case of fragmentation of partons with momentum $k$ into a hadron with momentum $P$ (a) and the spectator model description (b).

spin asymmetries at high energies. In order to understand the basic features of these matrix elements we perform a spectral analysis by modeling the distribution and fragmentation functions under reasonable assumptions.

For the correlators, depicted in Figs. 1(a) and 2(a), one has expressions in terms of matrix elements of bilocal operators that are frequently used as a starting point in modeling distribution and fragmentation functions. In particular the spectator model, pictorially given in Figs. 1(b) and 2(b), has become fairly popular, because it is easy, flexible and intuitively attractive. On the other hand, one should be very careful, because the predictive power depends on limiting oneself in the choice of spectator (e.g. a diquark with fixed mass in case of the nucleon) and using simple vertices. In fact making a spectral analysis of the spectator and allowing for the most general vertices one would lose all predictive power. Having said these words of caution, we will investigate in this paper differences between distribution and fragmentation functions using a spectral analysis and using physical intuition in restricting the momentum dependence and asymptotic behavior of the vertices. In this context, the relevant gluonic pole matrix elements that we want to study are $\Phi_{G}\left(k, k-k_{1}\right)$ and $\Delta_{G}\left(k, k-k_{1}\right)$ shown in Figs. 3 and 4 Of these matrix elements only the dependence on the collinear components $x$ and $x_{1}$ in the expansion of the momenta is needed. We find that while both $\Phi_{G}\left(x, x-x_{1}\right)$ and $\Delta_{G}\left(x, x-x_{1}\right)$ are nonzero, taking the limit $x_{1} \rightarrow x, \Phi_{G}(x, x)$ remains non-zero, while $\Delta_{G}(x, x)$ vanishes.

The vanishing of the T-odd gluonic pole matrix elements is important in the study of universality of TMD distribution functions (DFs) and fragmentation functions (FFs). For the collinear case T-symmetry can be used as a constraint on the parton correlators, limiting the DFs to T-even ones. Such a constraint does not apply for the fragmentation correlator because the final state hadron is part of a jet and as such not a plane wave, allowing both T-even and T-odd FFs. But for spin 0 and spin $1 / 2$ hadrons no T-odd functions appear at leading twist (leading order in the hard scale) [11, 12]. Including transverse momentum dependence, both the distribution and fragmentation correlators ( $\Phi$ and $\Delta)$ are no longer constrained by T-symmetry. The reason is that the appropriate color gauge invariant operators in the correlator, in particular the gauge links, are not T-invariant. For DFs this provides a mechanism leading to T-odd functions, such as the Sivers function [13]. But, for FFs there are now in principle two mechanisms leading to T-odd functions [14].

A nice feature, however, is that the two mechanisms leading to T-odd functions can be distinguished. The Todd operator structure can be traced back to the color gauge link that necessarily appears in correlators to render them color gauge-invariant. But the operator structure of the correlator is also a consequence of the necessary resummation of all contributions that arise from collinear gluon polarizations, i.e. those along the hadron momentum. How this resummation takes effect is a matter of calculation. The result is a process dependence in the path in the gauge link. After azimuthal weighting of cross sections one simply finds that the T-odd features originating from the gauge link lead to specific factors with which the T-odd functions appear in observables. Comparing T-odd effects in DFs in semi-inclusive deep inelastic scattering (SIDIS) and the Drell-Yan process one finds a relative minus sign [15]. Similarly, comparing T-odd effects in FFs in SIDIS and electron-positron annihilation one also finds a relative minus sign, at least for the T-odd effect originating from the operator structure (gauge link) 14]. The effect coming from the hadron-jet final state not being a plane wave will not lead to process dependent factors.

Before coming to the significance of our work, we need one more ingredient. We already mentioned that relating T-odd effects in different processes, requires azimuthal weighting, which projects out the transverse momentum weighted parts of the correlators $\Phi$ and $\Delta$, referred to as transverse moments $\Phi_{\partial}$ and $\Delta_{\partial}$, respectively. The T-odd operator parts are precisely the soft limits $\left(k_{1} \rightarrow 0\right)$ of the gluonic pole matrix elements [14]. Thus

$$
\begin{aligned}
& \Phi_{\partial}=\tilde{\Phi}_{\partial}+\pi \Phi_{G}\left(k_{1}=0\right), \\
& \Delta_{\partial}=\tilde{\Delta}_{\partial}+\pi \Delta_{G}\left(k_{1}=0\right),
\end{aligned}
$$

When $\Delta_{G}\left(k_{1}=0\right)$ is zero, there still are T-odd FFs contained in $\Delta_{\partial}$. They appear in the matrix elements of the T-even operator combination in $\tilde{\Delta}_{\partial}$ involving a hadronjet state (non-plane-wave) and they are process independent, for instance the T-odd Collins function [16]. In contrast T-odd DFs in $\Phi_{\partial}$ only can come from $\Phi_{G}\left(k_{1}=0\right)$. These DFs can still be universal but appear with calculable process dependent gluonic pole factors [17, 18]. 


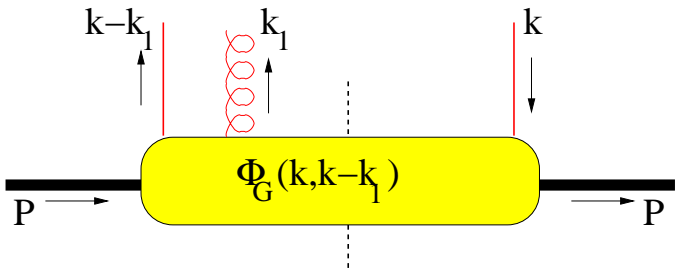

(a)

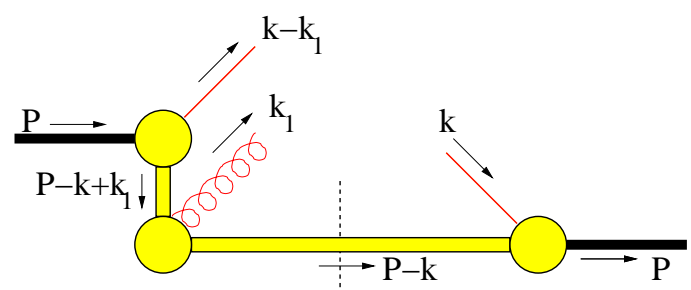

(b)

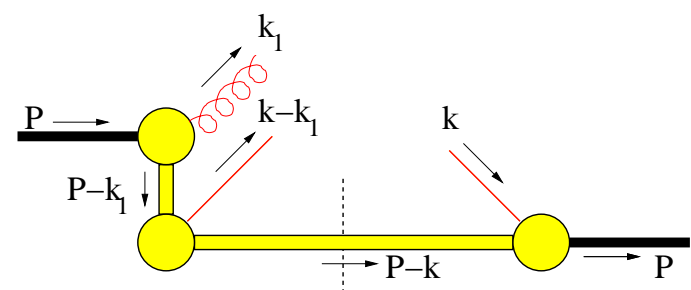

(c)

FIG. 3: The graphical representation of the quark-quarkgluon correlator $\Phi_{G}$ for the case of distributions including a gluon with momentum $k_{1}$ (a), and the possible intermediate states (b) and (c) in a spectator model description.

The above had already been shown in model calculations for SIDIS and $e^{+} e^{-}$annihilation [19, 20 and more recently in hadron-hadron scattering [21, 22]. In these calculations the authors look at the full process in the model, carefully studying the cuts, rather than concentrating on the soft part only. By contrast, we look at the soft part only, that is, the multi-parton correlators $\Phi_{G}$ and $\Delta_{G}$. In principle such an approach of only looking at the soft part is also possible starting with TMD dependent two-parton correlators $\Phi$ and $\Delta[23,24,25,26,27,28,29,30,31,32]$. To generate T-odd contributions for TMD functions in such an approach one needs to go at least to one-loop 33] performing a perturbative expansion on the gauge link [24, 25]. Extraction of the complete T-odd gluonic pole contribution is difficult. Our approach, starting directly with the color gauge invariant multi-parton correlator (that is having re-summed the gauge link [14]) has the advantage we can work with tree-level matrix elements and just perform a spectral analysis to extract the T-odd gluonic pole contributions.

In the next section we give details on the gluonic pole matrix elements, followed by the spectator model ap-

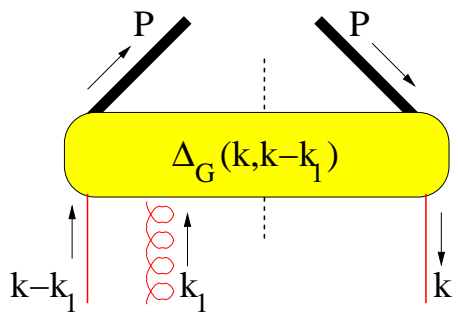

(a)

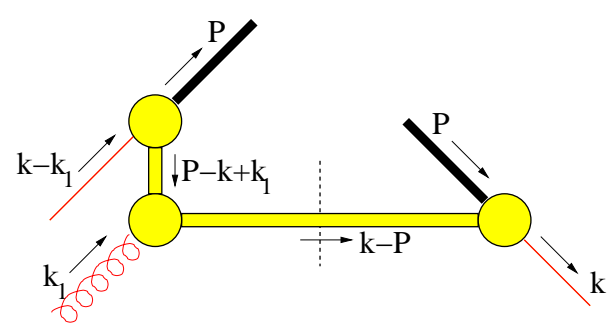

(b)

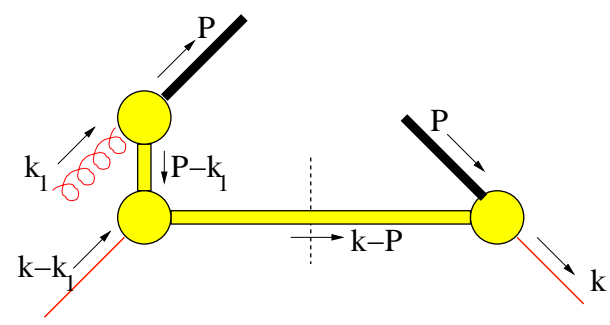

(c)

FIG. 4: The graphical representation of the quark-quarkgluon correlator $\Delta_{G}$ in the case of fragmentation including a gluon with momentum $k_{1}$ (a) and the possible intermediate states (b) and (c) in a spectator model description.

proach. In the concluding section, we outline the shortcomings of our approach and also discuss possibilities to use the approach for more detailed estimates for the Todd FFs.

\section{GLUONIC POLE MATRIX ELEMENTS}

At high energies, it is useful to make a Sudakov decomposition of the momenta of active partons, $k=$ $x P+\sigma n+k_{T}$. The Sudakov vector $n$ is an arbitrary light-like four-vector $n^{2}=0$ that has non-zero overlap $P \cdot n$ with the hadron's momentum $P$. We will simply choose $P \cdot n=1$. In a hard process, the Sudakov vector incorporates the presence of other momenta that are hard with respect to the hadron under consideration, e.g. $n \approx P^{\prime} / P \cdot P^{\prime}$. We can now also work with light-cone coordinates. Including mass effects one would have $n_{-}=n$ and $n_{+}=P-\frac{1}{2} M^{2} n$ and with $k^{ \pm} \equiv k \cdot n_{\mp}$ we have

$$
\begin{aligned}
& k^{+}=k \cdot n=x \\
& k^{-}=k \cdot P-\frac{1}{2} x M^{2}=\sigma+\frac{1}{2} x M^{2} .
\end{aligned}
$$


Vectors in the transverse plane can be obtained from the transverse projector, $g_{T}^{\mu \nu}=g^{\mu \nu}-n_{+}^{\{\mu} n_{-}^{\nu\}}$.

As the effects of the component $k^{-}$will appear suppressed by two powers of the hard scale as compared to the collinear term, it is integrated over and one considers quark-quark correlators on the light-front $(\mathrm{LF}: \xi \cdot n=0)$

$$
\begin{aligned}
\Phi_{i j}^{[\mathcal{U}]}\left(x, k_{T}\right)= & \int \frac{d(\xi \cdot P) d^{2} \xi_{T}}{(2 \pi)^{3}} e^{i k \cdot \xi} \\
& \left.\times\left\langle P\left|\bar{\psi}_{j}(0) \mathcal{U}_{[0 ; \xi]} \psi_{i}(\xi)\right| P\right\rangle\right\rfloor_{\mathrm{LF}} .
\end{aligned}
$$

The Wilson line or gauge link $\mathcal{U}_{[\eta ; \xi]}=$ $\mathcal{P} \exp \left[-i g \int_{C} d s \cdot A^{a}(s) t^{a}\right]$ is a path-ordered exponential along the integration path $C$ with endpoints at $\eta$ and $\xi$. Its presence in the hadronic matrix element is required by gauge-invariance. In the TMD correlator (5) the integration path $C$ in the gauge link is processdependent. In the diagrammatic approach the Wilson lines arise by resumming all gluon interactions between the soft and the hard partonic parts of the hadronic process [14, 34, 35, 36].

Collinear quark distribution functions are obtained from the TMD correlator after integration over $p_{T}$,

$$
\begin{aligned}
\Phi(x) & =\int d^{2} k_{T} \Phi^{[\mathcal{U}]}\left(x, k_{T}\right) \\
& \left.=\int \frac{d(\xi \cdot P)}{2 \pi} e^{i x \xi \cdot P}\left\langle P\left|\bar{\psi}(0) \mathcal{U}_{[0 ; \xi]}^{n} \psi(\xi)\right| P\right\rangle\right\rfloor_{\mathrm{LC}} .
\end{aligned}
$$

The non-locality is restricted to the light-cone (LC: $\xi \cdot n=$ $\left.\xi_{T}=0\right)$ and the gauge link is unique, being the straightline path along $n$. In azimuthal asymmetries one needs the transverse moments contained in the correlator

$$
\Phi_{\partial}^{\alpha[\mathcal{U}]}(x)=\int d^{2} k_{T} k_{T}^{\alpha} \Phi^{[\mathcal{U}]}\left(x, k_{T}\right)
$$

The TMD correlator, expanded in distribution functions depending on $x$ and $k_{T}^{2}$ contains T-even and T-odd functions, since the correlator is not T-invariant, which is attributed to the gauge link that depending on the process, accounts for specific initial and/or final state interactions depending on the color flow in the process. For the collinear case, the link structure becomes unique in the case of integration over $k_{T}$ (Eq. 6). For spin 0 and spin $1 / 2$ the quark and gluon correlators that appear at leading order in high energy processes contain only Teven operator combinations. Evaluated between plane waves one only finds T-even functions depending on $x$ in the parameterization.

For the collinear weighted case, the transverse moments in Eq. (7) one retains a nontrivial link-dependence that prohibits the use of T-invariance as a constraint. It is possible, however, to decompose the weighted quark (and also gluon) correlators as

$$
\Phi_{\partial}^{\alpha[\mathcal{U}]}(x)=\tilde{\Phi}_{\partial}^{\alpha}(x)+C_{G}^{[\mathcal{U}]} \pi \Phi_{G}^{\alpha}(x, x),
$$

with calculable process-dependent gluonic pole factors $C_{G}^{[\mathcal{U}]}$ and process (link) independent correlators $\tilde{\Phi}_{\partial}$ and $\Phi_{G}$. The correlator $\Phi_{\partial}$ contains the T-even operator combination, while $\Phi_{G}$ contains the T-odd operator combination. The latter is precisely the soft limit $x_{1} \rightarrow 0$ of a quark-gluon correlator $\Phi_{G}\left(x, x_{1}\right)$ of the type

$$
\left.\Phi_{G}^{\alpha}\left(x, x-x_{1}\right)=n_{\mu} \int \frac{d(\xi \cdot P)}{2 \pi} \frac{d(\eta \cdot P)}{2 \pi} e^{i x_{1}(\eta \cdot P)} e^{i\left(x-x_{1}\right)(\xi \cdot P)}\left\langle P\left|\bar{\psi}(0) U_{[0 ; \eta]}^{n} g G^{\mu \alpha}(\eta) U_{[\eta ; \xi]}^{n} \psi(\xi)\right| P\right\rangle\right\rfloor_{\mathrm{LC}},
$$

The universal T-odd distribution functions in the parameterization of $\Phi_{G}(x, x)$ appear in T-odd observables such as single spin asymmetries with the specific gluonic pole factors from Eq. 8 ,

The situation for fragmentation functions is different. The TMD fragmentation correlator depending on the collinear and transverse components of the quark momentum, $k=\frac{1}{z} P+k_{T}+\sigma n$, is given by [14]

$$
\Delta_{i j}^{[\mathcal{U}]}\left(z, k_{T}\right)=\left.\sum_{X} \int \frac{d\left(\xi \cdot P_{h}\right) d^{2} \xi_{T}}{(2 \pi)^{3}} e^{i k \cdot \xi}\left\langle 0\left|\mathcal{U}_{[0, \xi]} \psi_{i}(\xi)\right| P, X\right\rangle\left\langle P, X\left|\bar{\psi}_{j}(0)\right| 0\right\rangle\right|_{L F} .
$$

The collinear, $k_{T}$-integrated correlator

$$
\Delta(z)=\int d^{2} k_{T} \Delta^{[\mathcal{U}]}\left(z, k_{T}\right)=\left.\sum_{X} \int \frac{d(\xi \cdot P)}{2 \pi} e^{i z^{-1}(\xi \cdot P)}\left\langle 0\left|\mathcal{U}_{[0, \xi]}^{n} \psi_{i}(\xi)\right| P, X\right\rangle\left\langle P, X\left|\bar{\psi}_{j}(0)\right| 0\right\rangle\right|_{L C},
$$

only contains a T-even operator combination. Nevertheless one could in principle have T-even and T-odd fragmentation functions depending on $z$ since the hadronic state $|P, X\rangle$ is an out-state, which is not T-invariant. For spin 0 and spin $1 / 2$ hadrons no T-odd function appear at leading twist because of other constraints. At sub-leading twist they do appear [37].

In the transverse moments obtained after $k_{T}$-weighting,

$$
\Delta_{\partial}^{\alpha}[\mathcal{U}](z)=\int d^{2} k_{T} k_{T}^{\alpha} \Delta^{[\mathcal{U}]}\left(z, k_{T}\right)=\tilde{\Delta}_{\partial}^{\alpha}\left(\frac{1}{z}\right)+C_{G}^{[\mathcal{U}]} \pi \Delta_{G}^{\alpha}\left(\frac{1}{z}, \frac{1}{z}\right) .
$$


the two link independent correlators $\tilde{\Delta}_{\partial}$ and $\Delta_{G}$ contain again a T-even and T-odd operator combination, respectively. The gluonic pole correlator is again the soft limit, $z_{1}^{-1}=x_{1} \rightarrow 0$, of the quark-gluon correlator

$$
\Delta_{G i j}^{\alpha}\left(x, x-x_{1}\right)=\left.\sum_{X} \int \frac{d(\xi \cdot P)}{2 \pi} \frac{d(\eta \cdot P)}{2 \pi} e^{i x_{1}(\eta \cdot P)} e^{i\left(x-x_{1}\right)(\xi \cdot P)}\left\langle 0\left|\mathcal{U}_{[0, \eta]}^{n} g G^{n \alpha}(\eta) \mathcal{U}_{[\eta, \xi]}^{n} \psi_{i}(\xi)\right| P, X\right\rangle\left\langle P, X\left|\bar{\psi}_{j}(0)\right| 0\right\rangle\right|_{L C} .
$$

Because of the appearance of hadronic states $|P, X\rangle$, each of correlators in Eq. 12 contains in principle T-even and T-odd functions. Rather than having a doubling of Todd functions, we will show in a spectator model approach that $\Delta_{G}(x, x)=0$, which implies that T-odd fragmentation functions in the transverse moments only come from $\tilde{\Delta}_{\partial}$, which appear with a universal strength (no gluonic pole factors). We will show this in a spectator approach starting with the collinear quark-gluon correlators in Eqs. 9 and 13 rather than the model approaches $[23,24,25,26,27,28,29,30,31,32]$ that looked at the transverse momentum dependent quark correlators in Eqs 5 and 10

\section{THE SPECTATOR MODEL APPROACH}

In a typical spectator model approach to distribution or fragmentation correlators one considers a spectator with mass $M_{s}$. The result for the cut, but untruncated, diagrams, such as in Figs. 1 and 2 are of the form

$\Phi\left(x, k_{T}\right) \sim \int d(k \cdot P) \frac{F\left(k^{2}, k \cdot P\right)}{\left(k^{2}-m^{2}+i \epsilon\right)^{2}} \delta\left((k-P)^{2}-M_{s}^{2}\right)$,

where $F\left(k^{2}, k \cdot P\right)$ contains the numerators of propagators and/or traces of them in the presence of Dirac Gamma matrices, as well as the vertex form factors (see for example [38]). The explicit momenta, using light-cone coordinates $\left[p^{-}, p^{+}, p_{T}\right]$ as discussed in the beginning of the previous section, are

$$
\begin{aligned}
& P=\left[\frac{M^{2}}{2}, 1,0_{T}\right], \\
& P-k=\left[\frac{M_{s}^{2}-k_{T}^{2}}{2(1-x)}, 1-x,-k_{T}\right], \\
& k=\left[\frac{(1-x) M^{2}-M_{s}^{2}+k_{T}^{2}}{2(1-x)}, x, k_{T}\right] .
\end{aligned}
$$

In the above the delta function constraint in Eq. 14 has been implemented. One finds that the numerator $F\left(k^{2}, k \cdot P\right)=F\left(x, k_{T}^{2}\right)$ and hence

$$
\Phi\left(x, k_{T}\right) \sim \frac{(1-x)^{2} F\left(x, k_{T}\right)}{\left(\mu^{2}(x)-k_{T}^{2}\right)^{2}}
$$

with

$$
\mu^{2}(x)=x M_{s}^{2}+(1-x) m^{2}-x(1-x) M^{2} .
$$

Note that $k_{T}^{2}=-\boldsymbol{k}_{T}^{2} \leq 0$. The details of the numerator function depend on the details of the model, including the vertices, polarization sums, etc. These must be chosen in such a way as to not produce unphysical effects, such as a decaying proton if $M \geq m+M_{s}$, thus $m$ in Eq. 14 must represent some constituent mass in the quark propagator, rather than the bare mass. The useful feature of the result in Eq. 18 is its ability to produce reasonable valence and even sea quark distributions using the freedom in the model connected to an intuitive picture. The results for the fragmentation function in the spectator model is identical upon the substitution of $x=1 / z$.

Next we turn to the same spectral analysis of the gluonic pole correlator using the picture given in Figs. 3 for distribution functions and the picture given in Figs. 4 for fragmentation functions. Again, we only need to investigate one of the cases. We parameterize the gluon momentum as

$$
k_{1}=\left[k_{1}^{-}, x_{1}, k_{1 T}\right]
$$

where $k_{1}^{-}=k_{1} \cdot P-\frac{1}{2} x_{1} M^{2}$ will be the first component to be integrated over. The relevant momenta (implementing the on-shell condition for $P-k$ ) are

$$
\begin{aligned}
k-k_{1} & =\left[-k_{1}^{-}+\frac{(1-x) M^{2}-M_{s}^{2}+k_{T}^{2}}{2(1-x)}, x-x_{1}, k_{T}-k_{1 T}\right], \\
P-k+k_{1} & =\left[k_{1}^{-}+\frac{M_{s}^{2}-k_{T}^{2}}{2(1-x)}, 1-x+x_{1},-k_{T}+k_{1 T}\right], \\
P-k_{1} & =\left[-k_{1}^{-}+\frac{M^{2}}{2}, 1-x_{1},-k_{1 T}\right] .
\end{aligned}
$$


The basic result for the quark-gluon correlators $\Phi_{G}\left(x, x-x_{1}, k_{T}, k_{T}-k_{1 T}\right)$ becomes

$$
\begin{aligned}
\Phi_{G} \sim \frac{1}{\left(k^{2}-m^{2}\right)}\left\{\int \frac{d k_{1}^{-}}{2 \pi i} \frac{F_{1}\left(k_{1}^{-}, x, x_{1}, k_{T}, k_{1 T}\right)}{\left(k_{1}^{2}-m_{1}^{2}+i \epsilon\right)\left(\left(k-k_{1}\right)^{2}-m^{2}+i \epsilon\right)\left(\left(P-k+k_{1}\right)^{2}-M_{s 1}^{2}+i \epsilon\right)}\right. \\
\left.+\int \frac{d k_{1}^{-}}{2 \pi i} \frac{F_{2}\left(k_{1}^{-}, x, x_{1}, k_{T}, k_{1 T}\right)}{\left(k_{1}^{2}-m_{1}^{2}+i \epsilon\right)\left(\left(k-k_{1}\right)^{2}-m^{2}+i \epsilon\right)\left(\left(P-k_{1}\right)^{2}-M_{s 2}^{2}+i \epsilon\right)}\right\} \\
\sim \frac{1-x}{\left(\mu^{2}-k_{T}^{2}\right)}\left\{\int \frac{d k_{1}^{-}}{2 \pi i} \frac{F_{1}\left(k_{1}^{-}, x, x_{1}, k_{T}^{2}, k_{1 T}^{2}\right)}{\left(x_{1} k_{1}^{-}-A_{1}+i \epsilon\right)\left(\left(x_{1}-x\right) k_{1}^{-}-A_{2}+i \epsilon\right)\left(\left(1-x+x_{1}\right) k_{1}^{-}-B_{1}+i \epsilon\right)}\right. \\
\left.+\int \frac{d k_{1}^{-}}{2 \pi i} \frac{F_{2}\left(k_{1}^{-}, x, x_{1}, k_{T}^{2}, k_{1 T}^{2}\right)}{\left(x_{1} k_{1}^{-}-A_{1}+i \epsilon\right)\left(\left(x_{1}-x\right) k_{1}^{-}-A_{2}+i \epsilon\right)\left(\left(x_{1}-1\right) k_{1}^{-}-B_{2}+i \epsilon\right)}\right\},
\end{aligned}
$$

where, as before, $F_{i}\left(k_{1}^{-}, x, x_{1}, k_{T}^{2}, k_{1 T}^{2}\right)$ contain numerators and vertex functions [24, 25]. We use the quantities,

$$
\begin{aligned}
& 2 A_{1}=m_{1}^{2}-k_{1 T}^{2}, \\
& 2 A_{2}=m^{2}-\left(x-x_{1}\right) M^{2}+\frac{x-x_{1}}{1-x}\left(M_{s}^{2}-k_{T}^{2}\right) \\
& \quad-\left(k_{T}-k_{1 T}\right)^{2} \\
& 2 B_{1}=M_{s 1}^{2}-\frac{1-x+x_{1}}{1-x}\left(M_{s}^{2}-k_{T}^{2}\right) \\
& \quad-\left(k_{T}-k_{1 T}\right)^{2} \\
& 2 B_{2}=M_{s 2}^{2}-\left(1-x_{1}\right) M^{2}-k_{1 T}^{2} .
\end{aligned}
$$

These quantities depend on spectator masses, momentum fractions $\left(x\right.$ and $\left.x_{1}\right)$ and the transverse momenta $\left(k_{T}\right.$ and $k_{1 T}$ ). Besides the spectator mass $M_{s}$, two additional spectator masses $M_{s 1}$ and $M_{s 2}$ appear (see Figs. 3(b) and (c) or Figs. 4(b) and (c) and compare with the starting expression in Eq. 241). The quantity $\mu^{2}$ is the same one as given in Eq. 19. Assuming that the numerator does not grow with $k_{1}^{-}$one can easily perform the $k_{1}^{-}$integrations. We will for simplicity assume that the $F_{i}$ are independent of $k_{1}^{-}$, in which case we obtain for $x \geq x_{1} \geq 0$ (see Appendix),

$$
\begin{aligned}
& \Phi_{G}\left(x, x-x_{1}\right)=\int d^{2} k_{T} d^{2} k_{1 T} \\
& \left\{\frac{(1-x) F_{1}\left(x, x_{1}, k_{T}, k_{1 T}\right)}{\left(\mu^{2}-k_{T}^{2}\right)\left(x A_{1}+x_{1}\left(A_{2}-A_{1}\right)\right)\left(x_{1}\left(B_{1}-A_{2}\right)-x B_{1}-(1-x) A_{2}\right)\left((1-x) A_{1}+x_{1}\left(A_{1}-B_{1}\right)\right)}\right. \\
& \times\left[\left(x_{1}\left(B_{1}-A_{2}\right)-x B_{1}-(1-x) A_{2}\right) x_{1} \theta\left(x_{1}\right)\right. \\
& +\left((1-x) A_{1}+x_{1}\left(A_{1}-B_{1}\right)\right)\left(x_{1}-x\right) \theta\left(x_{1}-x\right) \\
& \left.+\left(x A_{1}+x_{1}\left(A_{2}-A_{1}\right)\right)\left(1-x+x_{1}\right) \theta\left(1-x+x_{1}\right)\right] \\
& +\frac{(1-x) F_{2}\left(x, x_{1}, k_{T}, k_{1 T}\right)}{\left(\mu^{2}-k_{T}^{2}\right)\left(x A_{1}+x_{1}\left(A_{2}-A_{1}\right)\right)\left(A_{2}-x B_{2}+x_{1}\left(B_{2}-A_{2}\right)\right)\left(x_{1}\left(A_{1}-B_{2}\right)-A_{1}\right)} \\
& \times\left[\left(A_{2}-x B_{2}+x_{1}\left(B_{2}-A_{2}\right)\right) x_{1} \theta\left(x_{1}\right)\right. \\
& +\left(x_{1}\left(A_{1}-B_{2}\right)-A_{1}\right)\left(x_{1}-x\right) \theta\left(x_{1}-x\right) \\
& \left.\left.+\left(x A_{1}+x_{1}\left(A_{2}-A_{1}\right)\right)\left(x_{1}-1\right) \theta\left(x_{1}-1\right)\right]\right\}
\end{aligned}
$$

Taking the limit $x_{1} \rightarrow 0$ we get the gluonic pole correlators, for distribution functions $(0 \leq x \leq 1)$,

$$
\Phi_{G}(x, x)=-\int d^{2} k_{T} d^{2} k_{1 T} \frac{(1-x) F_{1}\left(x, 0, k_{T}, k_{1 T}\right) \theta(1-x)}{\left(\mu^{2}-k_{T}^{2}\right)\left(x B_{1}+(1-x) A_{2}\right) A_{1}},
$$


and for fragmentation functions $(x=1 / z \geq 1)$

$$
\Delta_{G}(x, x)=0 .
$$

We note that this result depends on the assumption that the numerator does not grow with $k_{1}^{-}$. If this is the case one would find integrals of the type $\Theta_{111}^{1}$ (also given in the Appendix) rather than those of the type $\Theta_{111}^{0}$ and one does not get the required $x_{1} \theta\left(x_{1}\right)$ behavior in the calculation. In models, terms proportional to $k_{1}^{-} \sim k_{1} \cdot P$ may easily arise from numerators of fermionic propagators [32, 39] which in turn may easily be suppressed by form factors at the vertices. To prove a proper behavior within QCD one would need to study the fully unintegrated correlators such as e.g. in Ref. [40] and show that they fall off sufficiently fast as a function of $k_{1} \cdot P$.

\section{CONCLUSIONS}

In this work, we have investigated the gluonic pole contributions to the distribution and fragmentation functions. Instead of doing a quantitative analysis involving details of a phenomenological model, we limit ourselves to a spectral analysis within the spectator framework, in order to understand the basic features of these quantities. The advantage is that we are able to investigate only the soft parts at tree level and take the zero momentum limit of the gluon involved. We simply assume that masses and vertices do not spoil our analysis, which implies limits on the mass distributions of the spectators, use of vertices that cancel the bare-mass poles in the quark and gluon propagators and behavior of vertices that assures sufficient convergence of integrations. We find that under realistic assumptions, the gluonic pole contributions for fragmentation correlators vanish whereas these contributions do not vanish for distribution correlators. The result for fragmentation correlators at nonzero gluon momentum is nonzero. We stress that this certainly is not yet the full proof that gluonic pole matrix elements vanish in the case of fragmentation. However, we consider this analysis as a step towards such a proof and the possible direction to obtain such a proof by only considering the appropriate color gauge-invariant soft matrix elements. Such a proof is important as it eliminates a whole class of matrix elements parameterized in terms of T-odd fragmentation functions besides the T-odd fragmentation functions in the parameterization of the two-parton correlators. For instance, only one of the contributions to the spin asymmetries considered for jet-hyperon production in Ref. [41] remains. Moreover,the remaining fragmentation functions appear with the standard partonic cross section, so no gluonic pole cross sections need to be considered for fragmenting final-state partons, limiting these considerations to the distribution functions involving initial-state partons.

As mentioned, the results in this paper may point the way to find a full proof of $\Delta_{G}(x, x)=0$. The approach taken here to look at tree-level three-parton correlators, also can be used for explicit model calculations for Todd distribution functions originating from gluonic pole matrix elements and the investigation of their effects in single spin asymmetries.

\section{Acknowledgments}

This work was initiated at the WHEPP-X workshop in Chennai (January 2008). We thank Daniel Boer for useful discussions. L.G. acknowledges support from U.S. Department of Energy under contract DE-FG0207ER41460.

\section{APPENDIX A: USEFUL INTEGRALS}

Often it is useful to attack integrals containing propagator poles via light-cone variables, leading to integrals of the type

$$
\Theta_{n_{1} n_{2} \ldots}^{m}\left(x_{1}, x_{2}, \ldots\right)=\int \frac{d \alpha}{2 \pi i} \frac{\alpha^{m}}{\left(\alpha x_{1}-1+i \epsilon\right)^{n_{1}}\left(\alpha x_{2}-1+i \epsilon\right)^{n_{2}} \ldots},
$$

for which easy reduction rules exist [42]. We need specifically

$$
\begin{aligned}
& \Theta_{11}^{0}\left(x_{1}, x_{2}\right)=\frac{\theta\left(x_{1}\right) \theta\left(-x_{2}\right)-\theta\left(-x_{1}\right) \theta\left(x_{2}\right)}{x_{1}-x_{2}}=\frac{\theta\left(x_{1}\right)-\theta\left(x_{2}\right)}{x_{1}-x_{2}}, \\
& \begin{aligned}
\Theta_{111}^{0}\left(x_{1}, x_{2}, x_{3}\right) & =\frac{x_{2}}{\left(x_{1}-x_{2}\right)} \Theta_{11}^{0}\left(x_{2}, x_{3}\right)-\frac{x_{1}}{\left(x_{1}-x_{2}\right)} \Theta_{11}^{0}\left(x_{1}, x_{3}\right) \\
= & \frac{\left(x_{2}-x_{3}\right) x_{1} \theta\left(x_{1}\right)+\left(x_{3}-x_{1}\right) x_{2} \theta\left(x_{2}\right)+\left(x_{1}-x_{2}\right) x_{3} \theta\left(x_{3}\right)}{\left(x_{1}-x_{2}\right)\left(x_{2}-x_{3}\right)\left(x_{3}-x_{1}\right)}, \\
\Theta_{111}^{1}\left(x_{1}, x_{2}, x_{3}\right) & =\frac{1}{\left(x_{1}-x_{2}\right)}\left[\Theta_{11}^{0}\left(x_{2}, x_{3}\right)-\Theta_{11}^{0}\left(x_{1}, x_{3}\right)\right] \\
= & \frac{\left(x_{2}-x_{3}\right) \theta\left(x_{1}\right)+\left(x_{3}-x_{1}\right) \theta\left(x_{2}\right)+\left(x_{1}-x_{2}\right) \theta\left(x_{3}\right)}{\left(x_{1}-x_{2}\right)\left(x_{2}-x_{3}\right)\left(x_{3}-x_{1}\right)} .
\end{aligned}
\end{aligned}
$$


Including arbitrary pole positions, one finds

$$
\begin{aligned}
\int \frac{d \alpha}{2 \pi i} & \frac{1}{\left(\alpha x_{1}-A_{1}+i \epsilon\right)\left(\alpha x_{2}-A_{2}+i \epsilon\right)} \\
& =\frac{x_{1}-x_{2}}{x_{1} A_{2}-x_{2} A_{1}} \Theta_{11}^{0}\left(x_{1}, x_{2}\right) \\
& =\frac{\theta\left(x_{1}\right)-\theta\left(x_{2}\right)}{x_{1} A_{2}-x_{2} A_{1}}=\frac{\theta\left(x_{1}\right) \theta\left(-x_{2}\right)-\theta\left(-x_{1}\right) \theta\left(x_{2}\right)}{x_{1} A_{2}-x_{2} A_{1}}, \\
\int \frac{d \alpha}{2 \pi i} & \frac{1}{\left(\alpha x_{1}-A_{1}+i \epsilon\right)\left(\alpha x_{2}-A_{2}+i \epsilon\right)\left(\alpha x_{1}-A_{3}+i \epsilon\right)} \\
& =\frac{1}{x_{1} A_{2}-x_{2} A_{1}}\left[x_{2} \frac{x_{2}-x_{3}}{x_{2} A_{3}-x_{3} A_{2}} \Theta_{11}^{0}\left(x_{2}, x_{3}\right)-x_{1} \frac{x_{1}-x_{3}}{x_{1} A_{3}-x_{3} A_{1}} \Theta_{11}^{0}\left(x_{1}, x_{3}\right)\right] \\
& =\frac{\left(x_{2} A_{3}-x_{3} A_{2}\right) x_{1} \theta\left(x_{1}\right)+\left(x_{3} A_{1}-x_{1} A_{3}\right) x_{2} \theta\left(x_{2}\right)+\left(x_{1} A_{2}-x_{2} A_{1}\right) x_{3} \theta\left(x_{3}\right)}{\left(x_{1} A_{2}-x_{2} A_{1}\right)\left(x_{2} A_{3}-x_{3} A_{2}\right)\left(x_{3} A_{1}-x_{1} A_{3}\right)}, \\
\int \frac{d \alpha}{2 \pi i} & \frac{\alpha}{\left(\alpha x_{1}-A_{1}+i \epsilon\right)\left(\alpha x_{2}-A_{2}+i \epsilon\right)\left(\alpha x_{1}-A_{3}+i \epsilon\right)} \\
& =\frac{1}{x_{1} A_{2}-x_{2} A_{1}}\left[A_{2} \frac{x_{2}-x_{3}}{x_{2} A_{3}-x_{3} A_{2}} \Theta_{11}^{0}\left(x_{2}, x_{3}\right)-A_{1} \frac{x_{1}-x_{3}}{x_{1} A_{3}-x_{3} A_{1}} \Theta_{11}^{0}\left(x_{1}, x_{3}\right)\right] \\
& =\frac{\left(x_{2} A_{3}-x_{3} A_{2}\right) \theta\left(x_{1}\right)+\left(x_{3} A_{1}-x_{1} A_{3}\right) \theta\left(x_{2}\right)+\left(x_{1} A_{2}-x_{2} A_{1}\right) \theta\left(x_{3}\right)}{\left(x_{1} A_{2}-x_{2} A_{1}\right)\left(x_{2} A_{3}-x_{3} A_{2}\right)\left(x_{3} A_{1}-x_{1} A_{3}\right)} .
\end{aligned}
$$

[1] J. C. Collins and D. E. Soper, Nucl. Phys. B194, 445 (1982).

[2] R. L. Jaffe and X.-D. Ji, Nucl. Phys. B375, 527 (1992).

[3] A. V. Efremov and O. V. Teryaev, Sov. J. Nucl. Phys. 36, 140 (1982).

[4] A. V. Efremov and O. V. Teryaev, Phys. Lett. B150, 383 (1985).

[5] J.-W. Qiu and G. Sterman, Phys. Rev. Lett. 67, 2264 (1991).

[6] J.-W. Qiu and G. Sterman, Nucl. Phys. B378, 52 (1992).

[7] J.-w. Qiu and G. Sterman, Phys. Rev. D59, 014004 (1999), hep-ph/9806356.

[8] Y. Kanazawa and Y. Koike, Phys. Lett. B478, 121 (2000), hep-ph/0001021.

[9] H. Eguchi, Y. Koike, and K. Tanaka, Nucl. Phys. B763, 198 (2007), hep-ph/0610314.

[10] Y. Koike and K. Tanaka, Phys. Lett. B646, 232 (2007), hep-ph/0612117.

[11] X.-D. Ji, Phys. Rev. D49, 114 (1994), hep-ph/9307235.

[12] A. Bacchetta and P. J. Mulders, Phys. Rev. D62, 114004 (2000), hep-ph/0007120.

[13] D. W. Sivers, Phys. Rev. D41, 83 (1990).

[14] D. Boer, P. J. Mulders, and F. Pijlman, Nucl. Phys. B667, 201 (2003), hep-ph/0303034.

[15] J. C. Collins, Phys. Lett. B536, 43 (2002), hep$\mathrm{ph} / 0204004$.

[16] J. C. Collins, Nucl. Phys. B396, 161 (1993), hep$\mathrm{ph} / 9208213$.

[17] C. J. Bomhof, P. J. Mulders, and F. Pijlman, Phys. Lett. B596, 277 (2004), hep-ph/0406099.

[18] C. J. Bomhof and P. J. Mulders, JHEP 02, 029 (2007), hep-ph/0609206.

[19] A. Metz, Phys. Lett. B549, 139 (2002).
[20] J. C. Collins and A. Metz, Phys. Rev. Lett. 93, 252001 (2004), hep-ph/0408249.

[21] F. Yuan, Phys. Rev. Lett. 100, 032003 (2008), arXiv:0709.3272 [hep-ph].

[22] F. Yuan (2008), arXiv:0801.3441 [hep-ph].

[23] A. Bacchetta, R. Kundu, A. Metz, and P. J. Mulders, Phys. Rev. D65, 094021 (2002), hep-ph/0201091.

[24] X.-d. Ji and F. Yuan, Phys. Lett. B543, 66 (2002), hep$\mathrm{ph} / 0206057$.

[25] L. P. Gamberg, G. R. Goldstein, and K. A. Oganessyan, Phys. Rev. D67, 071504 (2003), hep-ph/0301018.

[26] L. P. Gamberg, G. R. Goldstein, and K. A. Oganessyan, Phys. Rev. D68, 051501 (2003), hep-ph/0307139.

[27] A. Bacchetta, A. Metz, and J.-J. Yang, Phys. Lett. B574, 225 (2003), hep-ph/0307282.

[28] A. Bacchetta, A. Schaefer, and J.-J. Yang, Phys. Lett. B578, 109 (2004), hep-ph/0309246.

[29] Z. Lu and B.-Q. Ma, Phys. Rev. D70, 094044 (2004), hep-ph/0411043.

[30] D. Amrath, A. Bacchetta, and A. Metz, Phys. Rev. D71, 114018 (2005), hep-ph/0504124.

[31] A. Bacchetta, L. P. Gamberg, G. R. Goldstein, and A. Mukherjee, Phys. Lett. B659, 234 (2008), arXiv:0707.3372 [hep-ph].

[32] L. P. Gamberg, G. R. Goldstein, and M. Schlegel (2007), arXiv:0708.2580 [hep-ph].

[33] S. J. Brodsky, D. S. Hwang, and I. Schmidt, Phys. Lett. B530, 99 (2002), hep-ph/0201296.

[34] A. V. Efremov and A. V. Radyushkin, Theor. Math. Phys. 44, 774 (1981).

[35] D. Boer and P. J. Mulders, Nucl. Phys. B569, 505 (2000), hep-ph/9906223.

[36] A. V. Belitsky, X. Ji, and F. Yuan, Nucl. Phys. B656, 
165 (2003), hep-ph/0208038.

[37] R. L. Jaffe and X.-D. Ji, Phys. Rev. Lett. 71, 2547 (1993), hep-ph/9307329.

[38] R. Jakob, P. J. Mulders, and J. Rodrigues, Nucl. Phys. A626, 937 (1997), hep-ph/9704335.

[39] L. P. Gamberg, D. S. Hwang, A. Metz, and M. Schlegel, Phys. Lett. B639, 508 (2006), hep-ph/0604022.
[40] J. C. Collins, T. C. Rogers, and A. M. Stasto (2007), arXiv:0708.2833 [hep-ph].

[41] D. Boer, C. J. Bomhof, D. S. Hwang, and P. J. Mulders, Phys. Lett. B659, 127 (2008), arXiv:0709.1087 [hep-ph].

[42] A. V. Belitsky (1997), hep-ph/9703432. 